\title{
Enhancement of Mast Cell Degranulation Mediated by Purinergic Receptor Activation and $\operatorname{PI3K}(\delta)$
}

\author{
Haruhisa Nishi ${ }^{1}$, Francois Niyonsaba ${ }^{2,3}$, Amir Pelleg $^{4}$, and Edward Schulman ${ }^{5}$ \\ ${ }^{1}$ Jikei University School of Medicine \\ ${ }^{2}$ Juntendo University \\ ${ }^{3}$ Juntendo University Graduate School of Medicine \\ ${ }^{4}$ Danmir Therapeutics \\ ${ }^{5}$ Drexel University College of Medicine
}

August 17, 2020

\begin{abstract}
Background: Mast cells express multiple metabotropic purinergic receptor (P2YR) subtypes, however, only few studies have evaluated their role in human mast cells (HMC) allergic response as measured by degranulation resulting from FceRI-activation. We have previously shown that extracellular nucleotides modify the FceRI-activation-dependent degranulation in HMC derived from human lungs, but the mechanism of this action has not been fully delineated. The present study was undertaken to determine the mechanism of P2YR's activation on HMC's degranulation and elucidate the specific post-receptor mechanistic steps/pathways involved. Methods: Sensitized LAD2 cells, a human derived mast cell line, were subjected to a weak allergic stimulation (WAS) using a low concentration of antigen in the absence and presence of the P2Y11R agonist, ATPrS. Results: In the presence of ATP $\gamma \mathrm{S}$, WAS-induced degranulation was enhanced by 7 -fold $(\mathrm{N}=4, p<0.01)$. None of the other P2YR agonists tested, including high concentrations of $\operatorname{ATP\gamma S}(1000 \mu \mathrm{M})$, enhanced WAS-induced intracellular $\mathrm{Ca}^{2+}$ mobilization, which is an important component of degranulation. Both a phosphoinositide 3-kinase (PI3K) inhibitor and the relevant gene knockout decreased the ATPrS-induced enhancement s of degranulation. ATPrS' effect was associated with enhanced phosphorylation of PI3K type $\delta(\mathrm{PI} 3 \mathrm{~K}(\delta))$ and protein kinase B (Akt), but not the phosphoinositide-dependent kinase-1 (PDK-1). The effects of ATPrS were dose dependently inhibited by NF157, a P2Y11R antagonist. Conclusion: We determined for the first time that

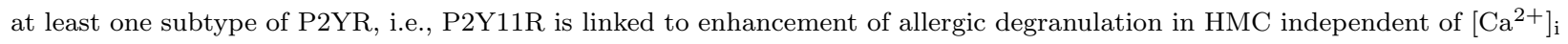
mobilization.
\end{abstract}

\section{Abstract}

Background: Mast cells express multiple metabotropic purinergic receptor (P2YR) subtypes, however, only few studies have evaluated their role in human mast cells (HMC) allergic response as measured by degranulation resulting from FceRI-activation. We have previously shown that extracellular nucleotides modify the FceRI-activation-dependent degranulation in HMC derived from human lungs, but the mechanism of this action has not been fully delineated. The present study was undertaken to determine the mechanism of P2YR's activation on HMC's degranulation and elucidate the specific post-receptor mechanistic steps/pathways involved.

Methods: Sensitized LAD2 cells, a human derived mast cell line, were subjected to a weak allergic stimulation (WAS) using a low concentration of antigen in the absence and presence of the P2Y11R agonist, ATPYS.

Results: In the presence of ATP $\gamma \mathrm{S}$, WAS-induced degranulation was enhanced by 7 -fold $(\mathrm{N}=4, p<0.01)$. None of the other P2YR agonists tested, including high concentrations of ATPrS $(1000 \mu \mathrm{M})$, enhanced 
WAS-induced intracellular $\mathrm{Ca}^{2+}$ mobilization, which is an important component of degranulation. Both a phosphoinositide 3-kinase (PI3K) inhibitor and the relevant gene knockout decreased the ATPrS-induced enhancement $\mathrm{s}$ of degranulation. ATP $\mathrm{S}$ ' effect was associated with enhanced phosphorylation of PI3K type $\delta(\operatorname{PI} 3 \mathrm{~K}(\delta))$ and protein kinase B (Akt), but not the phosphoinositide-dependent kinase-1 (PDK-1). The effects of ATP $\gamma \mathrm{S}$ were dose dependently inhibited by NF157, a P2Y11R antagonist.

Conclusion: We determined for the first time that at least one subtype of P2YR, i.e., P2Y11R is linked to enhancement of allergic degranulation in HMC independent of $\left[\mathrm{Ca}^{2+}\right]_{\mathrm{i}}$ mobilization.

\section{KEY WORDS}

allergic exacerbation, ATP $\mathrm{S}$, $\beta$-Hex release, $\mathrm{Ca}^{2+}$ mobilization, degranulation, FceRI, LAD2 mast cells, $\operatorname{PI3K}(\delta)$, P2Y11R.

Abbreviations used:

ATP $\gamma \mathrm{S}$ (adenosine 5'-(3-thio)triphosphate); $\beta$-Hex (beta-hexosaminidase); GAPDH (glyceraldehyde 3phosphate dehydrogenase); PDK-1 (Phosphoinositide-dependent kinase-1); PI3K( $\delta$ ) (phosphoinositide 3kinase type $\delta$ ); P2XR (purinergic receptor P2X ligand-gated ion channel); P2YR (metabotropic purinergic P2Y receptor); P2Y11R (metabotropic purinergic P2Y11 receptor), WAS (weak allergic stimulation).

\section{INTRODUCTION}

Mast cells, by virtue of their wide organ distribution, play important roles in both localized and systemic inflammatory processes and the host's immune response. ${ }^{1}$ Mast cells' degranulation, which follows the activation of the high affinity $\operatorname{IgE}$ receptor (FceRI), is associated with the release of cytokines, lipids, and chemical mediators of inflammation including histamine that can result in edema and pruritus via the type-I allergic response. ${ }^{2,3}$ In human subjects, these responses can not only decrease quality of life but may also be life-threatening. The function of human mast cells (HMC) in general and cultured human lung must cells (HLMC) in particular, during physiologic and pathophysiologic conditions have been the subject of multiple studies. ${ }^{4-7}$

Previously, we have reported that extracellular adenine nucleotides can either enhance or suppress allergic stimulation-induced degranulation (i.e., histamine release) in HLMC. ${ }^{8-10}$ Thus, we have hypothesized that the exacerbation of allergic symptoms could be mediated by the release of intracellular adenine nucleotides from cells associated with inflammatory processes. Indeed, it is well documented that adenosine 5'-triphosphate (ATP) is released from different types of cells under inflammatory conditions. ${ }^{11,12}$

Purinergic receptors are divided into two super families, the adenosine receptors P1R, which consists of four receptors: A1AdoR, A2aAdoR, A2bAdoR and A3AdoR, and P2R, the purine and pyrimidine receptors. The latter consists of two families: P2Y, the seven transmembrane domain G protein coupled receptors (metabotropic), and P2X, trans-cell membrane cationic channels (ionotropic); of which eight and seven receptors have been cloned, respectively, heretofore. ${ }^{13,14} \mathrm{P} 2 \mathrm{X} 7 \mathrm{R}$ plays a critical role in inflammatory processes. ${ }^{15,16} \mathrm{P} 2 \mathrm{YR}$ are expressed by mast cells, however, only one receptor, i.e., P2Y14R has been proposed to be linked to histamine release. ${ }^{17}$

In terms of endogenous extracellular nucleotides, ATP is the only ligand that activates both P2XR and P2YR. It is also known that ATP is the most potent agonist at P2Y11R site, while other P2YR subtypes are sensitive to other nucleotides such as ADP or uridine-derived nucleotides. 18,19 It is suggested that ATPrS as a stable, non-hydrolysable P2Y11R agonist mimics endogenous ATP's effects mediated by P2Y11R. ${ }^{18,19}$

The purpose of the present study was to investigate the mechanism of enhancement of a weak allergic stimulation (WAS)-induced degranulation in HMC following the activation of P2YR in LAD2 cells. ${ }^{20-22}$

$2 \mid$ METHODS

2.1 Cell culture and quantification of degranulation 
The LAD2 cell line isolated from the human bone marrow was a gift from Dr. Arnold Kirshenbaum (National Institutes of Health, National Institute of Allergy and Infectious Diseases, Bethesda, MD). ${ }^{23}$ These cells were grown in StemPro-34 media (Invitrogen, Carlsbad, CA), supplemented with $2 \mathrm{mM}$ L-glutamine, $100 \mathrm{IU} / \mathrm{ml}$ penicillin and $50 \mu \mathrm{g} / \mathrm{ml}$ streptomycin (Meiji Seika, Tokyo, Japan), and $100 \mathrm{ng} / \mathrm{ml}$ human stem cell factor (SCF) (Wako, Osaka, Japan). LAD2 cells were sensitized with anti-4-hydroxy-3-nitrophenylacetyl antibody (anti-NP-IgE, $1 \mu \mathrm{g} / \mathrm{mL}$ ) (Bio-Rad, Hercules, CA) or untreated (control) for $24 \mathrm{~h}$. They were then washed twice with PBS and once with calcium-free Tyrode's solution. WAS challenge was carried out in Tyrode's solution $\left(2 \mu \mathrm{M} \mathrm{Ca}^{2+}\right)$ with a concentration of NP-BSA of $0.13 \mu \mathrm{g} / \mathrm{mL}$ (Biosearch Technologies, Hoddesdon, UK) which was just above a threshold for release, for $20 \mathrm{~min}$ at $37^{\circ} \mathrm{C}$, sedimented and supernatants collected. Cells were similarly challenged with addition of $\mathrm{P} 1 \mathrm{R}$ and $\mathrm{P} 2 \mathrm{R}$ subtype selective agonists (i.e., adenosine, ATP, ADP, UTP, UDP, 2Me-S-ATP and ATP $\gamma \mathrm{S}$ ). In other experiments, cells were pre-treated with the Compound 15e (Fujifilm Wako, Osaka, Japan), a PI3K-inhibitor, and then antigen or control challenged in the absence and presence of ATP $\gamma \mathrm{S}$. The extent of degranulation was quantified using the granule component beta-hexosaminidase ( $\beta$-Hex) and was determined spectrophotometrically. The quantity of $\beta$-Hex released was determined by measurement of spectrophotometric absorbance at $405 \mathrm{~nm}$, after the addition of N-acetylbeta-glucosaminide $(4 \mathrm{mM})$, a substrate of $\beta$-Hex, to the supernatant and incubation at $37^{\circ} \mathrm{C}$ for $90 \mathrm{~min}$ followed by addition of glycine $(0.2 \mathrm{M})$. Total $\beta$-Hex content of the cells was determined by $1 \%$ Triton-X treatment.

$2.2 \mid \mathrm{Ca}^{2+}$ assay

For adherence, LAD2 cells were cultured on fibronectin $(40 \mu \mathrm{g} / \mathrm{mL})$-treated cover slips in StemPro-34 medium. Cells were loaded with fura2-AM $(2 \mu \mathrm{M})$, and fluorescence was monitored at an emission wavelength of $510 \mathrm{~nm} \cdot\left[\mathrm{Ca}^{2+}\right]_{\mathrm{i}}$ was expressed as the ratio of the $510 \mathrm{~nm}$ fluorescence intensity excited at $340 \mathrm{~nm}$ and at $380 \mathrm{~nm}\left(\mathrm{I}_{340} / \mathrm{I}_{380}\right)$. To evaluate calcium mobilization caused by various stimuli, cells treated with WAS or untreated (control) were challenged with calcium ionophore A23187, nucleotides, or a nucleoside in Tyrode's solution $\left(2 \mu \mathrm{M} \mathrm{Ca}^{2+}\right)$. In experiments designed to examine calcium flux, cells treated with antiNP-IgE or untreated (control) were exposed to calcium ionophore (A23187) (Cayman Chemical, Ann Arbor, $\mathrm{MI})$, nucleotides, or a nucleoside in Tyrode's solution $\left(2 \mu \mathrm{M} \mathrm{Ca}^{2+}\right)$ for $20 \mathrm{~min}$, followed by stimulation with NP-BSA at $37^{\circ} \mathrm{C}$ for $30 \mathrm{~min}$ or no stimulation

\section{3 | Western blots}

Proteins were extracted from LAD2 cells (from different cell stocks based on the difference of the seeding time and culture periods) by RIPA buffer containing both protease inhibitors (GE Healthcare, Uppsala, Sweden) and phosphatase inhibitors (Roche, Mannheim, Germany). The extracts were centrifuged at 34,500 $\mathrm{x} g$ for $10 \mathrm{~min}$ and the supernatants were run on polyacrylamide gels. Antibodies for P2X7R, P2Y11R, ectonucleotide triphosphate dephosphorylase 1 (NTPD-1 = CD39), ecto-5'-nucleotidase (CD73), PI3K, PDK1, Akt, phosphorylated (phos)-PI3K, phos-PDK-1, phos-Akt, and GAPDH were purchased from Abcam (Cambridge, UK), GeneTex (Irvine, CA), and Cell Signaling (Danvers, MA). HRP-conjugated secondary antibodies (Rockland, Gilbertsville, PA) were used to detect the 1st antibody signals by $\mathrm{H}_{2} \mathrm{O}_{2}$-induced color development on PVDF membranes. Western blotting images were quantitated by ImageJ (NIH, MD).

\subsection{RNA interference}

The short hairpin RNA (shRNA), including the "loop" and constructs for PI3K( $\delta$ )-knockdown, were constructed as follows:

Top strand:

5'-CACCGGTAATTGAACCAGTAGGCACGAATGCCTACTGGTTCAATTAC-3'

Bottom strand:

5'AAAAGTAATTGAACCAGTAGGCATTCGTGCCTACTGGTTCAATTACC-3' 
These oligo DNAs were annealed to form double-stranded DNA for insertion into pENTR/U6 (Invitrogen, Carlsbad, CA), a shRNA plasmid. The plasmid was amplified in the One Shot TOP10 Chemically Competent E. coli (Invitrogen, Carlsbad, CA). The plasmid was then extracted from the E. coli and purified for RNAi.

\section{5 | Transfection}

The shRNA plasmid was transfected into LAD2 cells using a MicroPorator (Digital Bio, Seoul, Korea) at $1800 \mathrm{~V}-20$ msec pulse. Following transfection, the cells were cultured in StemPro-34, without antibiotics for three days prior to use in experiments. LAD2 cells were co-transfected with plasmids containing either the shRNA constructs for PI3K $(\delta)$ mRNA knock-down or eGFP expression as previously described. ${ }^{24}$ The eGFP expression was used for the transfection's success rate and availability of the cells. LAD2 cells transfected only with the eGFP plasmid were used as control for effects of non-knockdown in both Western blot analysis and functional assays.

\subsection{Statistics}

Data were analyzed by one-way analysis of variance (ANOVA) followed by Tukey-Kramer post-hoc tests to reduce any false positive or type I errors. $p<0.05$ was considered to be statistically significant.

\section{3 | RESULTS}

\subsection{ATP $\gamma \mathrm{S}$ enhanced WAS-induced degranulation in LAD2 cells}

Three P1R (A2aAdo, A2bAdo, and A3Ado), two P2XR (P2X1 and P2X7), and five P2YR subtypes (P2Y1, P2Y6, P2Y11, P2Y12, and P2Y14) are expressed on LAD2 cells SUPPLEMENTAL FIGURE 1). Concerning these results, we next examined the cell responses to nucleotide and nucleoside. The ability of multiple selective purinergic receptor agonists (adenosine, ATP, ADP, UTP, UDP, 2Me-S-ATP, and ATP $\mathrm{S}$ ) at physiological concentrations $(1-100 \mu \mathrm{M})$ to induce degranulation in non-sensitized LAD2 cells was assessed. None of the P2YR agonists tested significantly induced degranulation (Figure 1A). In sensitized cells, WAS-induced release was significant vs. the spontaneous release of the non-stimulated controls. Moreover, ATP $\mathrm{SS}$ markedly enhanced WAS-induced degranulation in sensitized LAD2 cells (7-fold, $\mathrm{N}=4, p$ $<0.01)$ (Figure 1B). Even higher concentrations $(1000 \mu \mathrm{M})$ of $\operatorname{ATP} \gamma \mathrm{S}$ alone did not induce degranulation in non-sensitized LAD2 cells (Figure 1C), while the same high concentration of BzATP, a P2X7R agonist, which is known to activate many types of mast cells, induced degranulation in non-sensitized LAD2 cells (Figure 1C). The potency of high concentration $(1 \mathrm{mM})$ of BzATP's effect on degranulation was the same as that of the calcium ionophore (A23187; $2 \mu \mathrm{M}$ ), which is known to induce degranulation in mast cells ${ }^{25,} 26$. These data strongly suggested that P2Y11 was the best candidate for the observed WAS-enhancing effects, as ATP $\gamma \mathrm{S}$ acts is a known agonist at that receptor. Also, the lack of WAS degranulation- enhancing effects of UTP and ADP excluded P2Y1, P2Y6, P2Y12, and P2Y14. Further supporting the role of P2Y11 is its known coupling to both $\mathrm{G}_{\mathrm{q} / 11}$ and $\mathrm{G}_{\mathrm{s}}$ proteins that are known to be linked to induction and suppression, respectively, of mast cell degranulation. The enhancing effect by ATP $\gamma \mathrm{S}(100 \mu \mathrm{M})$ on degranulation was suppressed by NF157, a selective P2Y11R antagonist in a concentration-dependent manner (Figure 1D).

3.2 ATP $\mathrm{S}$ S does not affect $\mathrm{Ca}^{2+}$ mobilization enhancement in sensitized WAS-LAD2 cells

High allergic stimulation (spike) (Figure 2A) but not WAS (without spike) (Figure 2B) induced intracellular $\mathrm{Ca}^{2+}$ mobilization in sensitized LAD2 cells. ATP $\mathrm{S} S(10-1000 \mu \mathrm{M})$ alone induced moderate intracellular $\mathrm{Ca}^{2+}$ mobilization (Figure 2C), however, it did not significantly affect subsequent WAS-induced $\mathrm{Ca}^{2+}$ mobilization (Figure 2D) though strongly influenced degranulation (Figure 1B). From the results of degranulation assays (Figure 1) and intracellular $\mathrm{Ca}^{2+}$ mobilization (Figure 2), and expression of mRNA/protein of P2YR (SUPPLEMENTAL FIGURE 1), we then confirmed that P2Y11 was the receptor responsible for the enhancement of WAS-induced degranulation.

\subsection{Inhibition of PI3K suppressed the effects of ATP $\mathrm{SS}$ on WAS-induced degranulation in LAD2 cells}

The effects of inhibition of phosphoinositide 3-kinase (PI3K) were examined in sensitized LAD2 cells. LAD2 cells were co-transfected with plasmids that contained either the shRNA constructs for PI3K $(\delta)$ mRNA 
knock-down or contained eGFP alone (expression over 85\%). PI3K expression was suppressed 50\% vs. the cells without the PI3K(ס) mRNA knock-down. (Figure 3A). The PI3K inhibitor compound 15e suppressed ATP ${ }_{\gamma}$ S's enhancement of WAS-induced degranulation in a concentration dependent manner (Fig, 3C). Also, the shRNA-transfected and control plasmid-transfected cells were challenged with anti-NP IgE. In the shRNA-transfected LAD2 cells, ATP $\gamma \mathrm{S}(100 \mu \mathrm{M})$-induced enhancement of WAS-induced degranulation was inhibited significantly (Figure 3B).

3.4 ATPץS modulates the phosphorylation of intracellular signal proteins related to PI3K( $\delta)$ in LAD2 cells

The effects of $\operatorname{ATP} \gamma \mathrm{S}$ on the phosphorylation of key kinases related to intracellular $\operatorname{PI} 3 \mathrm{~K}(\delta)$ activation cascade were determined. ATP $\gamma \mathrm{S}(100 \mu \mathrm{M})$ enhanced the phosphorylation of PI3K $(\delta)$ and Akt in LAD2 cells (Figure 4A). Comparison of signal levels in the images of PI3K $(\delta)$, phosphorylated PI3K $(\delta)$ (phosPI3K(ס)), PDK-1, phos-PDK-1, Akt, and phos-Akt are shown in Figure 4B. These data indicate that both PI3K(ס) and Akt were phosphorylated by ATP $\gamma \mathrm{S}$ and are up regulated by WAS; especially in case of Akt. However, PDK-1 which is known to be a link between PI3K( $\delta)$ and Akt, was not phosphorylated.

3.5 Expression of ectonucleotidases in LAD2 cells

LAD2 cells express ectonucleoside triphosphate dephosphorylase-1 (CD39) (Figure 5A) but not ecto-5'nucleotidase (CD73) (Figure 5B), suggesting extracellular nucleotides could be degraded to adenosine 5'monophosphate (AMP) but not to adenosine. Thus, administrated or endogenous ATP could be broken-down to AMP (by CD39), but not to adenosine (absent CD73).

\section{4 | DISCUSSION}

In a prior report, we showed that extracellular ATP was a powerful enhancer of low level IgE-mediated degranulation in HLMC. ${ }^{9,10}$ The intracellular signal transduction mechanisms underlying these effects have not been fully delineated. This study is the first to examine the intracellular mechanisms of the enhancement of IgE-mediated degranulation by the activation of a P2YR in HMC. The present data indicate that P2Y11R is linked to degranulation responses in LAD2 cells. This conclusion is based on (i) the expression of P2Y11 (Supplemental Figure 1), (ii) the lack of enhancing effect of purines and pyrimidines that target the other P2YR expressed by LAD2 cells, (iii) the strong enhancing effects of ATP $\gamma \mathrm{S}$, an agonist at the P2Y11R, ${ }^{25-27}$ and (iv) the marked inhibition of ATP $\gamma \mathrm{S}$ ' effects by NF157, a selective P2Y11R antagonist. ${ }^{28,29}$

Mechanistically, the P2Y11R effects involve the PI3K/Akt pathway but not the induction of intracellular $\mathrm{Ca}^{2+}$ mobilization following WAS triggering. This is in congruence with previous reports on PI3K and mast cell activation. ${ }^{30,31}$ It is well known that increased intracellular levels of free $\mathrm{Ca}^{2+}$ plays a key role in mast cell activation leading to degranulation. ${ }^{32,33} \mathrm{IgE}$ receptor (FcعRI) activation is usually associated with intracellular $\mathrm{Ca}^{2+}$ mobilization. However, intracellular $\mathrm{Ca}^{2+}$-independent steps involving degranulation in mast cells have also been reported. ${ }^{34-37}$ We show here that the enhancement of WAS degranulation mediated by P2Y11R activation can be independent of $\mathrm{Ca}^{2+}$ mobilization (Figure 2) ; but in contrast it involves the PI3K/Akt pathway. This is supported by the dose-dependent inhibition of the ATP $\gamma \mathrm{S}$ ' enhancing effects

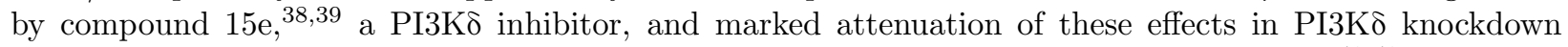
LAD2 cells. Though PDK-1 is known as one of the key elements of the PI3K/Akt pathway, ${ }^{40-42}$ the present results regarding phosphorylation of the intracellular signal proteins do not support involvement of PDK-1 in ATPrS-enhancement of WAS degranulation in LAD2 cells.

Aside from enhancement of allergic degranulation of mast cells, ${ }^{9,10}$ multiple phlogistic effects of ATP and adenosine in the lungs have been described. We have shown that extracellular (inhaled) ATP directly triggers a central pulmonary-pulmonary vagal reflex resulting in bronchoconstriction and cough. ${ }^{43}$ We have also suggested that ATP released from activated platelets plays a mechanistic role in syncope and bradycardia associated with pulmonary embolism. ${ }^{43,44}$ Furthermore, these findings are in congruence with the expression of purinergic receptors in the lungs ${ }^{45,46}$ mediating inflammatory reactions. In addition, adenosine monophosphate (AMP) has been used as a selective bronchoconstrictor in the assessment of airway hypersensitivity in asthmatic patients vs. non-asthmatics. ${ }^{44,47}$ Holgate et al. have shown that aerosolized AMP-induced 
bronchoconstriction in human subjects is mediated by adenosine acting on lung mast cells. ${ }^{48}$ However, under in vitro conditions, AMP can act as a nucleotide and not nucleoside (i.e., adenosine). In the current report we have shown that LAD2 cells lack CD73 required to degrade AMP to adenosine (Figure 5).

Accordingly, using primary cultured human lung mast cells (HLMC), from human pulmonary parenchymal tissues we have previously shown that AMP had no effect on mast cell degranulation. ${ }^{8-10}$ Thus, it seems that in vivo, ATP acts directly on HLMC but AMP's action in vivo depends on its degradation to adenosine by ecto-enzymes.

Physiologic agonist/agonist-receptor systems require a non-degraded agonist present at its cognate receptor site. The sensitivity to effects of purinergic agonists at purinergic receptor sites is thus influenced by the expression and activity of ectonucleotidases on cells' surface. ${ }^{49,50}$ It follows that the "right" purinergic agonist at the "right" purinergic cell surface receptor in the "right" permissive ectonucleotidases environment must "line up" in aggregate to result in effective WAS enhancement in the case of P2Y11 and LAD2 cells. This also means that higher cell surface expression of ectonucleotidases (e.g. CD39) might reduce allergic exacerbations because the enhanced degradation of ATP to AMP and the latter's lack of effect in the case of human lung mast cell degranulation. ${ }^{9,51}$

Previously, using another human cell line, we reported the half-life of extracellularly ATP/ADP. ${ }^{24}$ The cell line expressed the ectonucleotidases CD39, CD73, and alkaline phosphatase. In that study, the halflife of ATP $(1 \mathrm{mM})$ was $14.88 \mathrm{~min} .{ }^{24}$ Recent studies have emphasized the role of ectonucleotidases in the magnitude of ATP's effects in pulmonary and other organ disorders. ${ }^{52,53}$ It seems that many physiological factors interact to either maintain or degrade extracellular ATP in vivo, depending on localized physiological conditions such as $\mathrm{pH}$. It is plausible that data obtained in vitro may be optimized to achieve therapeutic advantages in clinical settings.

P2Y11R is coupled to $G_{q / 11^{-}}$but also to $G_{s}$ - protein $^{13}$ so that stimulation of P2Y11R could increase intracellular cAMP levels via the $\mathrm{G}_{\mathrm{s}}$ pathway. Accumulation of intracellular cAMP could activate PKA or the Epac (exchange protein directly activated by cAMP) system via the activation of adenylyl cyclase. ${ }^{54}$ However, cAMP is well known to suppress mast cell degranulation. 16 There are several reports that Epac1/2 can link to intracellular $\mathrm{Ca}^{2+}$ mobilization, ${ }^{55}$ but Epac1/2 do not cause mast cell activation. ${ }^{56}$ Our results suggest that the effect of ATP $\mathrm{SS}$ on WAS-induced degranulation in LAD2 cells is not related to the cAMP/Epac pathways (Supplemental Figure 2). Our study suggests that the WAS-induced degranulation, is by the P2Y11R- linkage to $G_{\mathrm{q} / 11}$ including the PI3K system and not Gs protein. Thus, the enhancing effect of $\operatorname{ATP} \gamma \mathrm{S}$ on WAS-induced degranulation in sensitized LAD2 is likely not directly related to intracellular $\mathrm{Ca}^{2+}$ mobilization.

The results of the present study indicate that P2Y11R is expressed in human mast cells. Its activation is linked to marked enhancement of WAS-induced degranulation. Induction of intracellular $\mathrm{Ca}^{2+}$ mobilization is not required for the P2Y11 effects on WAS. The enhancement of WAS via P2Y11R links to PI3K $(\delta)$ and Akt activation and not PDK-1. These results indicate that in the clinical setting given a favorable physiologic milieu, even a weak allergic trigger can lead to development of much heightened expression of IgE-mediated reactions and worsening allergic symptoms.

\section{ACKNOWLEDGEMENTS}

We thank Prof. Arnold Kirshenbaum for kindly supplying the LAD2 cells. HN is supported by The Jikei University Research Fund by a grant in The LIXIL Jyukankyo Foundation. ESS is supported by the Margaret Wolf Research Endowment.

\section{CONFLICT OF INTEREST}

The authors have no conflicting financial interest.

\section{AUTHOR CONTRIBUTION}


The potential role of purine nucleotides in the function of human lung mast cells under physiologic and pathophysiologic conditions was originally conceived by AP and ESS. HN conducted all experiments; he was assisted by FN. HN and AP cowrote the manuscript. ESS supervised the experiments and edited the manuscript. All authors have read the final draft of the manuscript and approved it submission for publication.

\section{SUPPORTING INFORMATION}

Additional supporting information may be found online in the Supporting Information section.

ORCID

Haruhisa NIshi: https://orcid.org/0000-0001-9056-7358

François Niyonsaba: https:// orcid.org/0000-0002-0588-8315

Amir Pelleg: https://orcid.org/

Edward S.Schulman: https://orcid.org/0000-0003-2928-2665

FIGURES

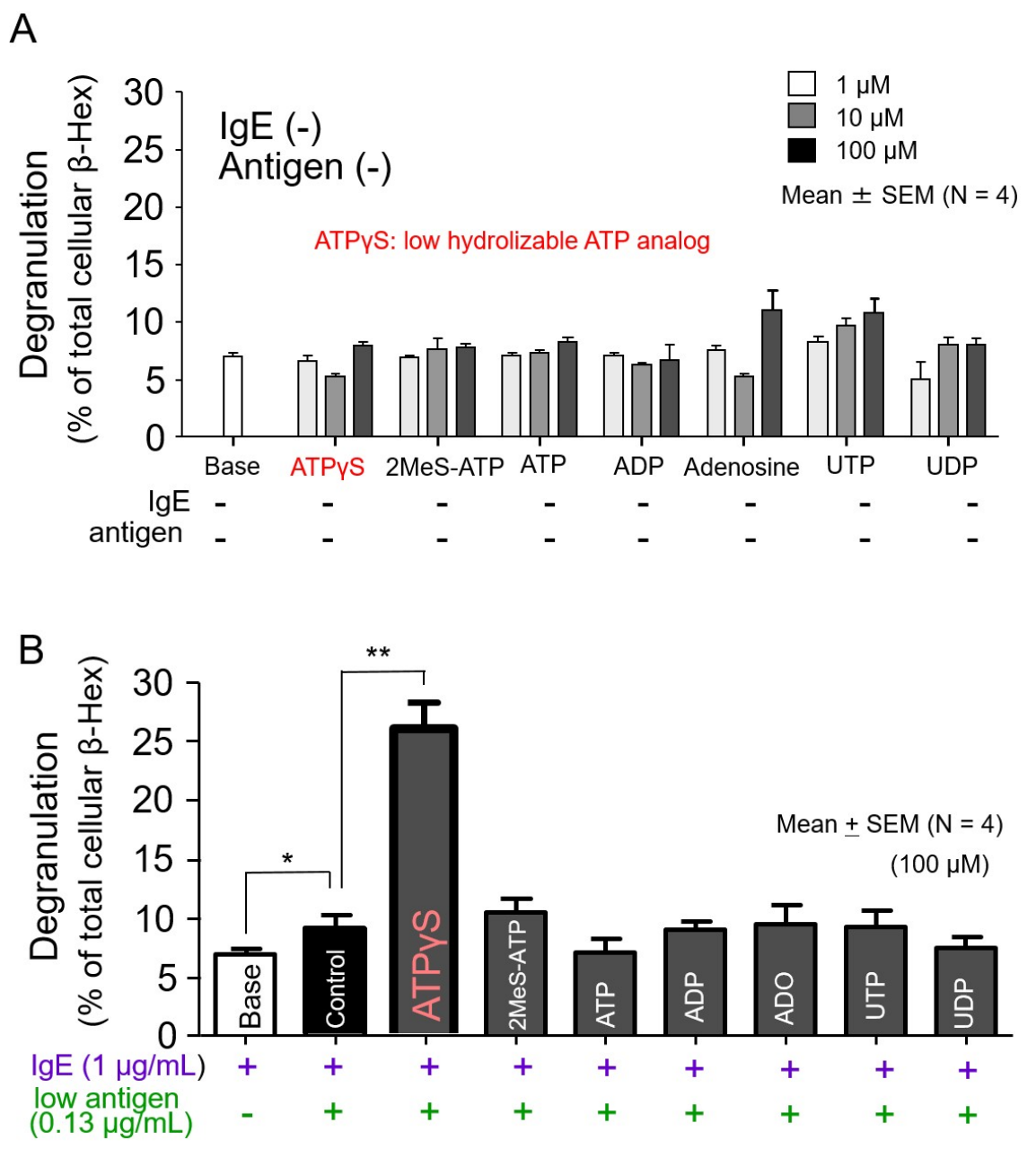


D (Sensitized LAD2)

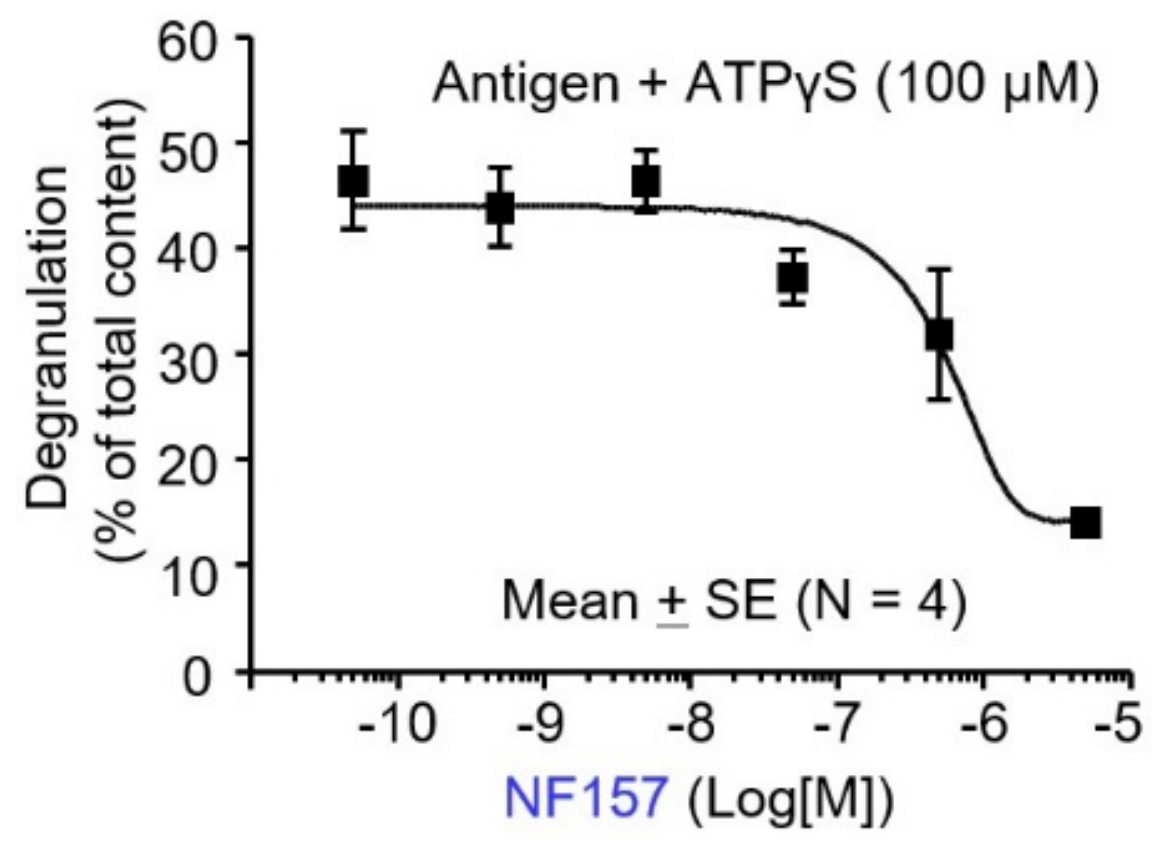




\section{(Non-sensitized LAD2)}

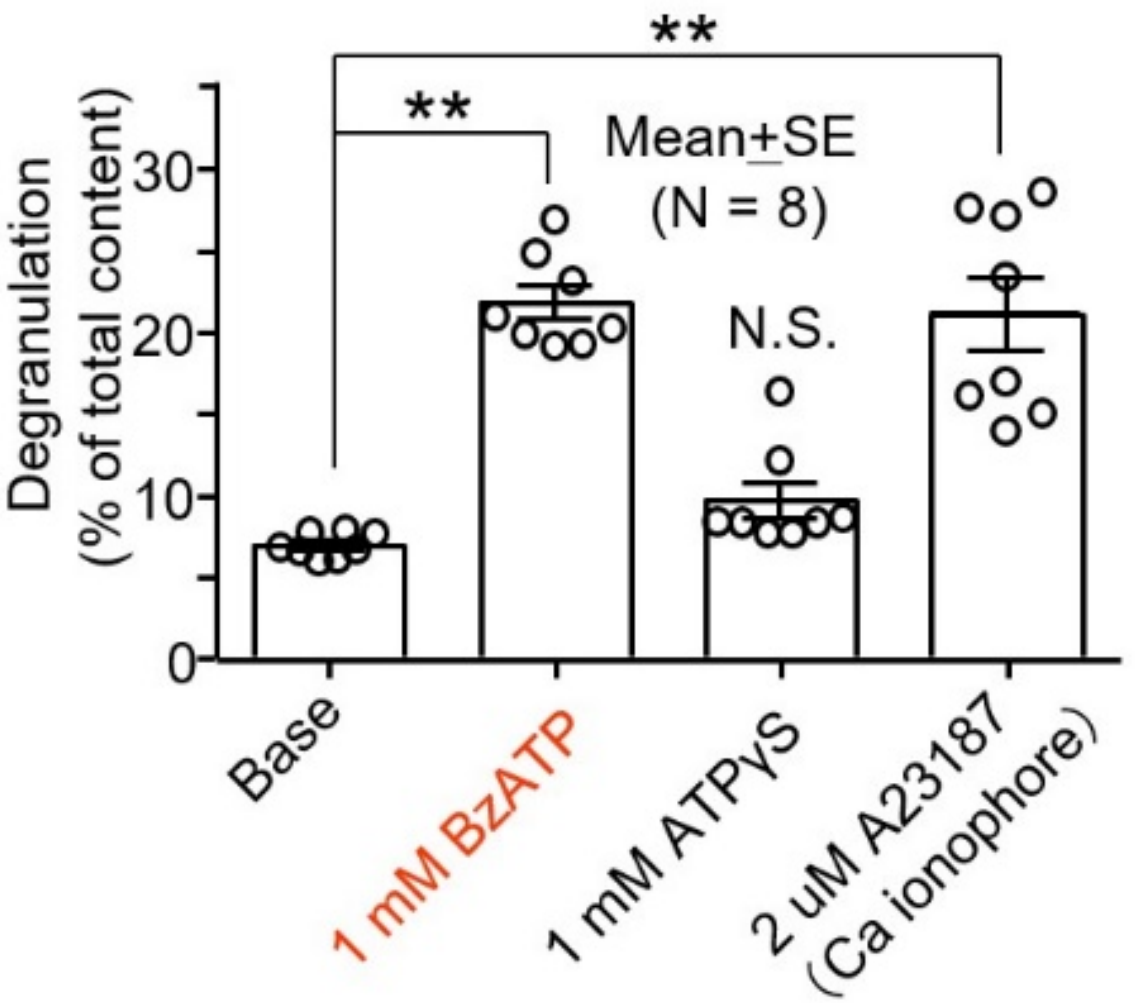

FIGURE 1 Degranulation ( $\beta$-Hex release) assay in non-sensitized/sensitized LAD2 cells. A: Analysis of degranulation in LAD2 cells induced by extracellular purines/pyrimidines (1-100 $\mu \mathrm{M})$. Each column shows the percentage of $\beta$-Hex-induced release vs. total cellular $\beta$-hex. Base represents the spontaneous release of the non-stimulated LAD2. B: Degranulation effects of extracellular purines/pyrimidines on weak (low) allergic stimulation induced by antigen NP-BSA $(0.13 \mu \mathrm{g} / \mathrm{mL})$ in anti-NP-IgE $(1 \mu \mathrm{g} / \mathrm{mL})$ sensitized LAD2 cells. C: Effects of high concentration $(1 \mathrm{mM})$ of BzATP (P2X7R agonist) and ATPrS (P2Y11R agonist). Each agonist was added alone to non-sensitized cells. The effect of A23187 $(2 \mu \mathrm{M})$, a $\mathrm{Ca}^{2+}$ ionophore, is shown as a comparison. D: The effect of NF157, a P2Y11R selective antagonist, on ATP $\gamma \mathrm{S}$ in anti-NP IgE sensitized LAD2 cells. The antagonist was added to the sensitized cells for 5 min prior to the addition of $\mathrm{ATP} \gamma \mathrm{S}$ and the antigen (NP-BSA). 


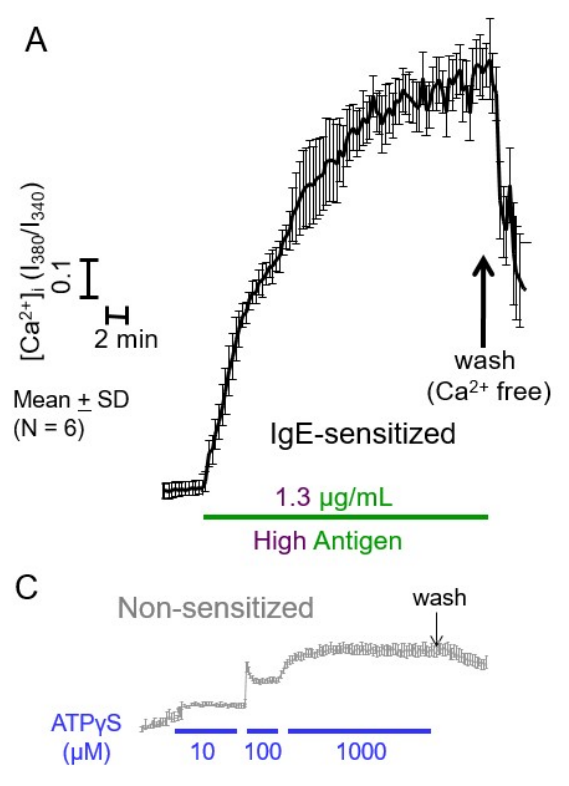

B

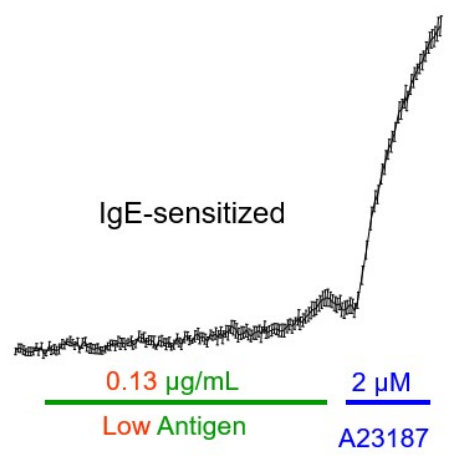

$\mathrm{D}$

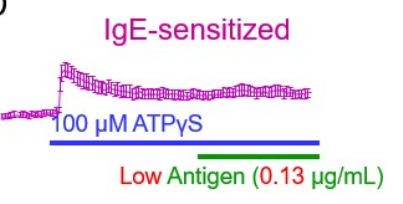

FIGURE 2 Effects of ATP $\gamma \mathrm{S}$ and/or a weak ("low") allergic stimulation on intracellular $\mathrm{Ca}^{2+}$ mobilization in LAD2 cells. A: High antigen-stimulation in anti-NP-IgE sensitized cells; B: Weak ("low") antigen stimulation in NP-IgE-sensitized cells; C: ATP $\gamma \mathrm{S}$ stimulation in non-sensitized cells; d: ATP $\gamma \mathrm{S}$ followed by weak ("low") antigen stimulation in sensitized cells. Colored-horizontal lines indicate the exposure periods of each ligand. In A, B, and D LAD2 cells were sensitized using anti-NP IgE $(1 \mu \mathrm{g} / \mathrm{mL})$ for 4 hrs at room temperature before the challenges. Each data set shown is Mean $\pm \mathrm{SD}(\mathrm{N}=6)$.
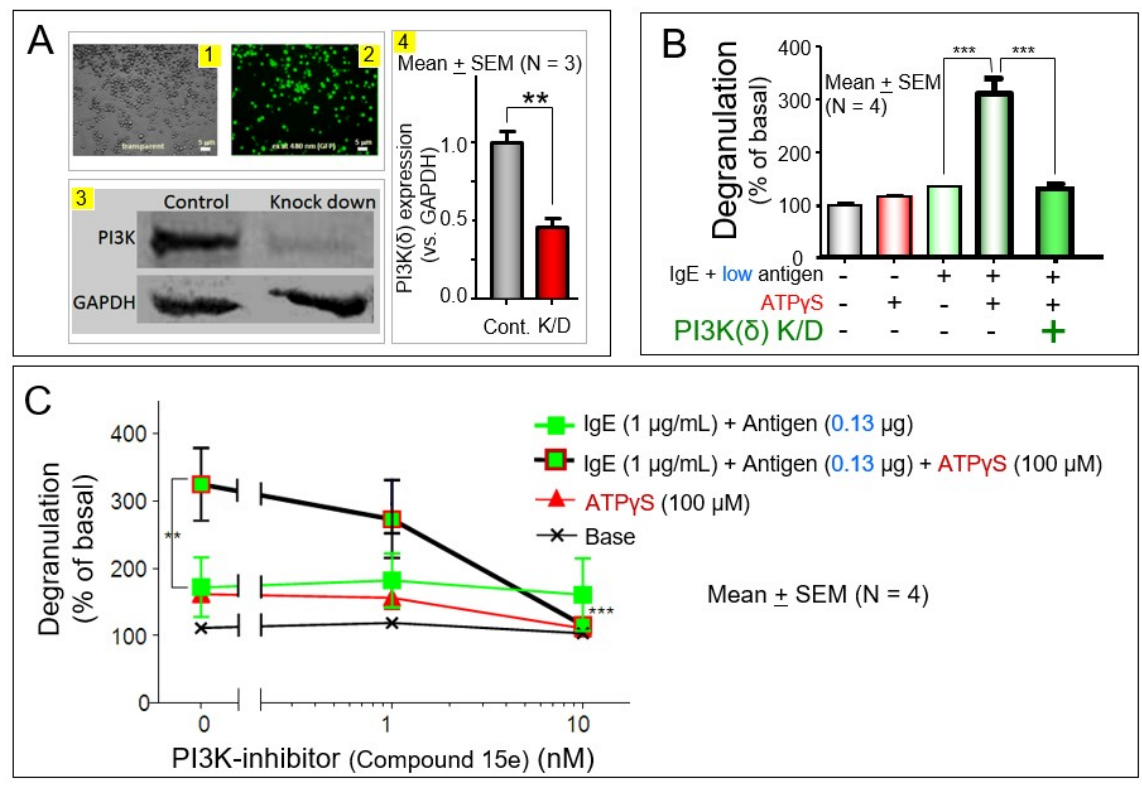

FIGURE 3 Relevancy of PI3K for ATPYS-induced enhancement of WAS-degranulation in LAD2. A-1: Light microscopy of LAD2 cells transfected with the PI3K knockdown construct and eGFP plasmid. A-2: The same field excited at $480 \mathrm{~nm}$. More than $85 \%$ of the transfected cells successfully expressed GFP signal. A-3: Western blots of the protein extracted control and transfected LAD2 cells. A-4: Comparison of the levels 
in the images of A-3. B: Effect of PI3K type $\delta(\mathrm{PI} 3 \mathrm{~K}(\delta))$ knockdown $(\mathrm{K} / \mathrm{D})$ on degranulation caused by ATP $\gamma$ S-induced allergic enhancement. C: Effect of PI3K-inhibitor on low weak allergic stimulation-induced degranulation in LAD2 cells. Each graph initially shows the percentage of $\beta$-Hex in non-stimulated cells (Base) with no PI3K-inhibitor present. PI3K-inhibitor was added 30 min before the stimulation. Mean \pm $\mathrm{SE}\left(\mathrm{N}=4\right.$, duplicate), ${ }^{* *} p<0.01$ (the differences based on the WAS-induced degranulation from $\left.15 \mathrm{e}\right),{ }^{* * *} p$ $<0.001$ (the differences based on the effects of $15 \mathrm{e}$ at 0 and $10 \mathrm{nM}$ ).

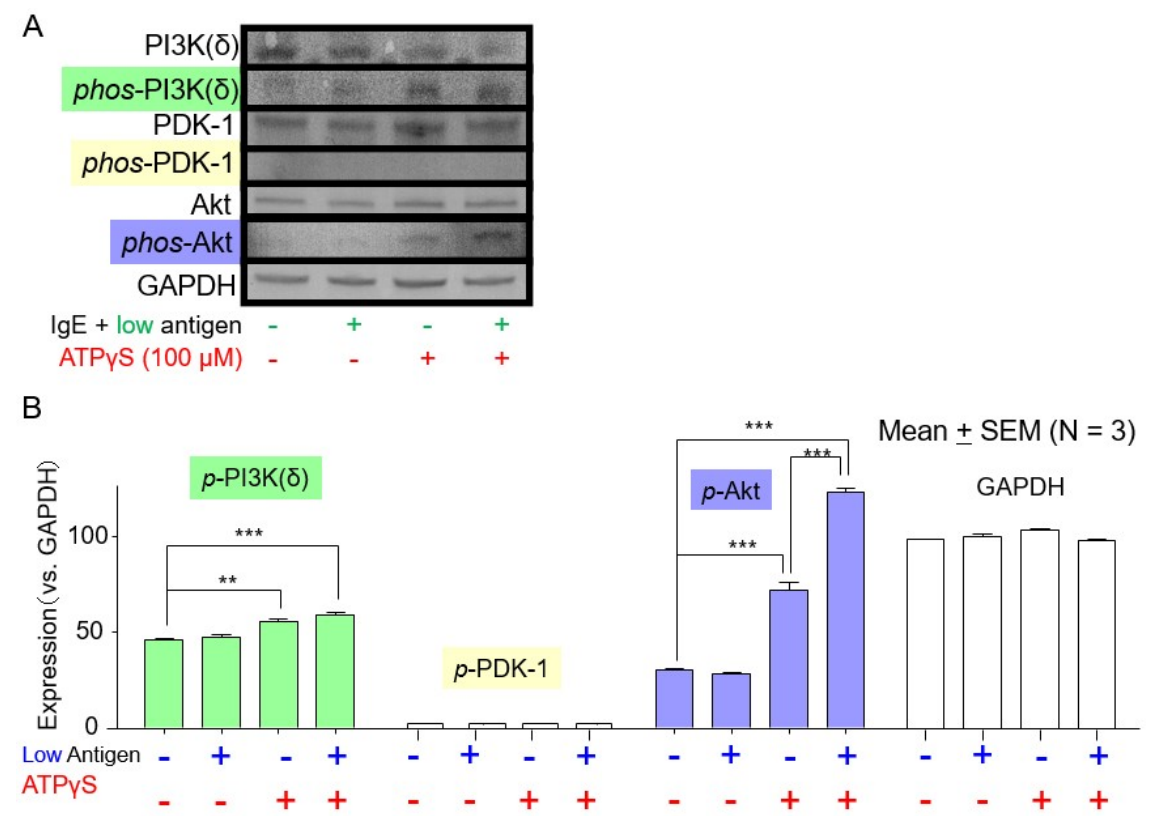

FIGURE 4 Effect of ATP $\gamma \mathrm{S}$ on the phosphorylation of intracellular kinases. Protein samples were extracted from LAD2 cells after challenges as shown. A: Images of non-phosphorylated and phosphorylated forms of kinases. B: Comparison of signal levels in the images of phos-PI3K, phos-PDK-1, and phos-Akt in A. "phos-" means phosphorylated proteins. Mean \pm SEM $(\mathrm{N}=5),{ }^{* *} p<0.01,{ }^{* * *} p<0.001$.

A

CD39

(NTPDase1)

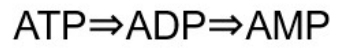

LAD2-1 LAD2-2 LAD2-3

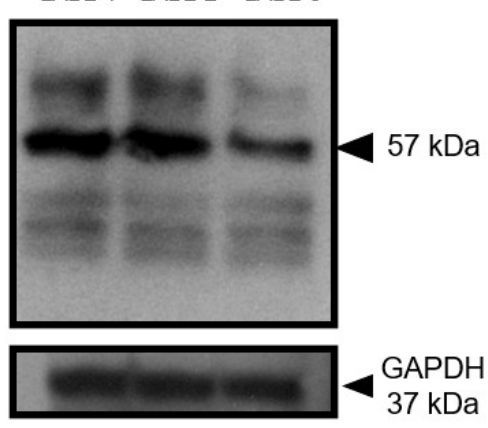

B

CD73

(Ecto-5'-nucleotidase)

$\mathrm{AMP} \Rightarrow$ Adenosine

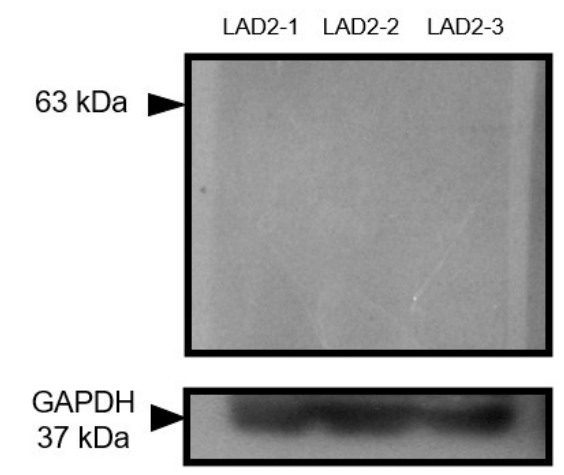

FIGURE 5 Expression of ectonucleotidases in three different lots of LAD2 cells. A: ectonucleoside triphos- 
phate diphosphohydrolase-1, also known as NTPDase1 or CD39; B: ecto-5'-nucleotidase, also known as 5'-nucleotidase (5'-NT) or CD73. CD39 which breaks-down ATP to AMP was shown to be expressed in all three lots of LAD2 cells tested, while CD73 which breaks down nucleotides to nucleosides (adenosine) was not expressed. Expression of GAPDH is also shown as a housekeeping gene protein.

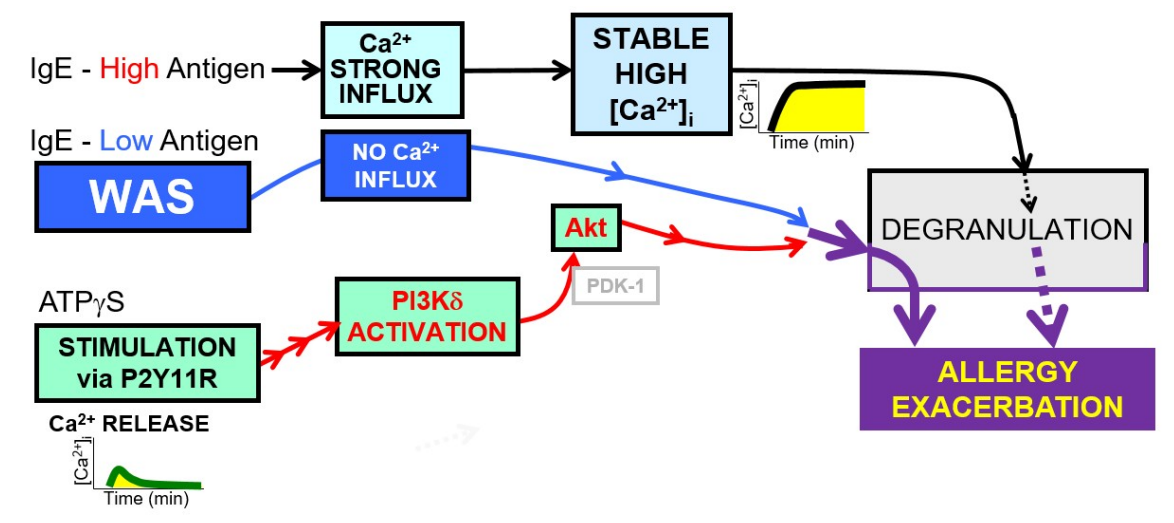

FIGURE 6 Schematic diagram of the present study suggests that the mast cell P2Y11R modulates the enzymatic cascade between PI3K $(\delta)$ and Akt, leading to enhancement of a weak FceRI-induced allergic stimulation/degranulation (WAS). Thus, P2Y11R, an endogenous purine (ATP) receptor, may strongly modify weak type I allergic responses.

\section{SUPPORTING INFORMATION}

Supplemental figure legends

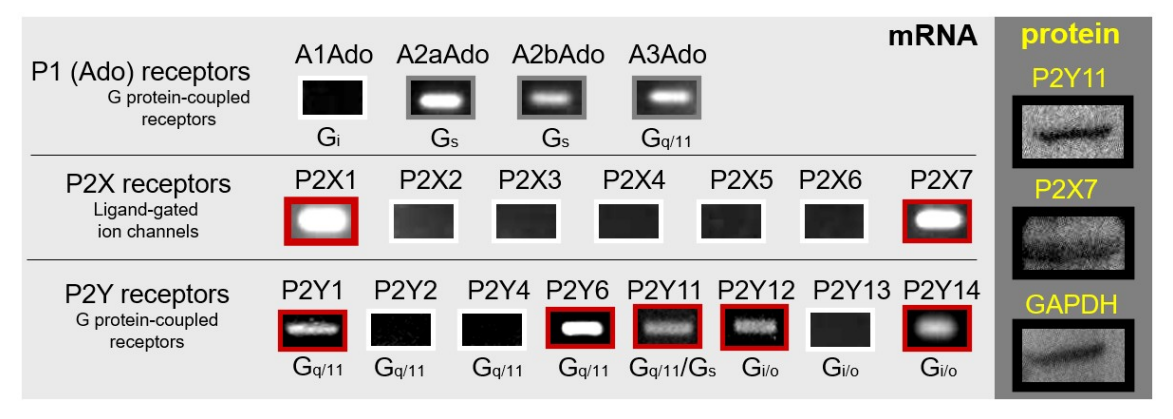

SUPPLEMENTAL FIGURE 1. Detection of mRNA and purinergic receptor protein. All RT-PCR procedures were initially performed with a gradient of eight annealing temperatures between 45 and $60^{\circ} \mathrm{C}$. All specific primer sets used for PCR amplification in the present study with expected molecular weight ware shown in the SUPPLEMENTAL TABLE 1. For the protein detection, western blots procedures were done with 1st antibodies for P2Y11R, P2X7R that were purchased from GeneTex (Irvine, CA). 

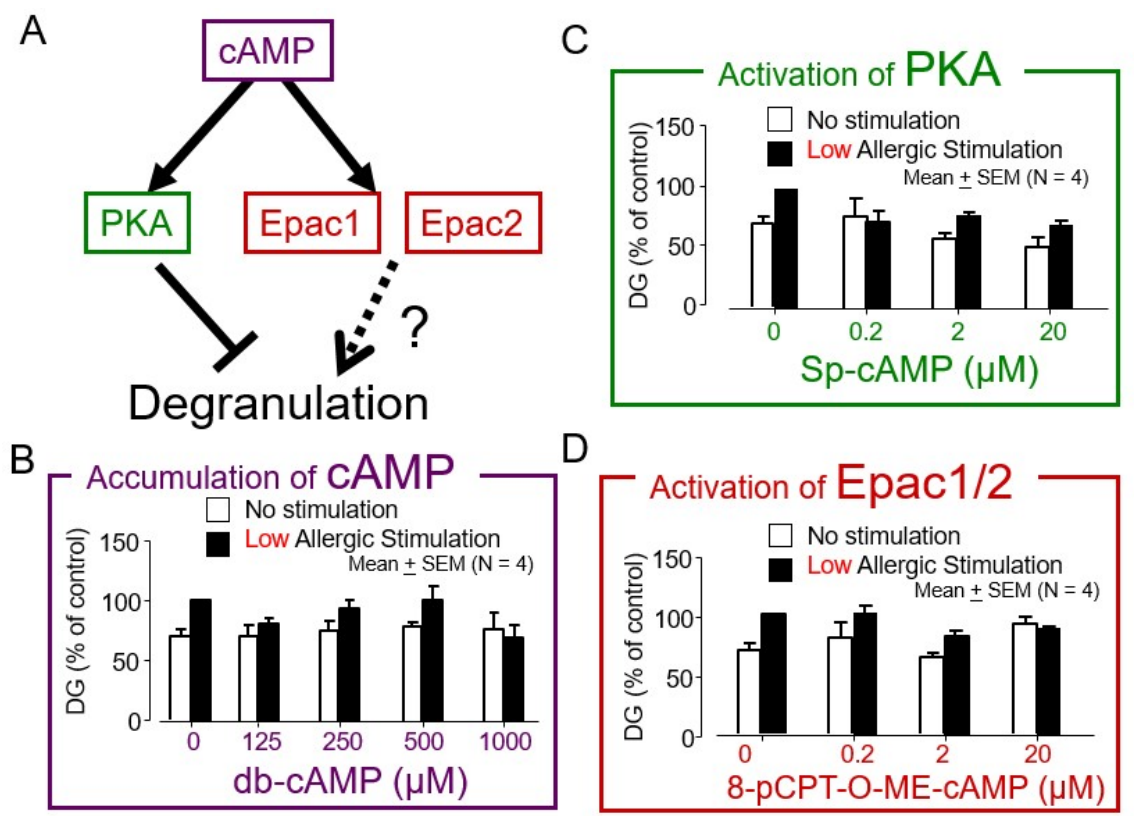

SUPPLEMENTAL FIGURE 2. Lack of enhancing effects of the principal elements of the cAMP pathway on weak allergic stimulation (WAS) in LAD2. A: A simple conceivable diagram of cAMP pathway with PKA, Epac1/2, and resultant effects on degranulation in mast cells. It is known that PKA inhibits degranulation; Epac is reported to link to PI3K-Akt pathway in some cells; B: Effect of dibutyryl-cyclic AMP (db-cAMP; cellular membrane permeable cyclic AMP); C: Sp-cAMP, a cell-permeable, potent, selective activator of cAMP-dependent protein kinase (PKA); D: and 8-pCPT-2'-O-Me-Cyclic AMP, an 8-(4-chlorophenylthio) analog of cAMP that activates Epac1/2 on weak allergic stimulation-induced low level of degranulation. No significant effects on WAS were seen in B, C, or D. Epac: Exchange protein directly activated by cAMP.

SUPPLEMENTAL TABLE 1

mRNA sequences size (bp)

A1Ado Sense 5'-CTCGCCATCCTCATCAACATT-3' 245

Antisense 5'-CAGCCAAACATAGGGGTCAGTC-3'

A2aAdo Sense 5'-GCCCCTCTCTGGCTCATGTACCTG-3' 450

Antisense 5'-TCATCAGGACACTCCTGCTCCATC-3'

A2bAdo Sense 5'-GAGCTGATGGACCACTCGAGG-3' 341

Antisense 5'-ACACCGAGAGCAGGCTGTACC-3'

A3Ado Sense 5'-AACGTGCTGGTCATCTGCGTGGTC-3' 441

Antisense 5'-GTAGTCCATTCTCATGACGGAAAC-3'

P2X1 Sense 5'-CTGTGAAGACGTGTGAGATCTTTGG-3' 463

Antisense 5'-TTGAAGAGGTGACGGTAGTTGGTC-3'

P2X2 Sense 5'-GCTGCTCATCCTGCTCTACTTCGTGTGG-3' 399

Antisense 5'-GGGGTAGTGGATGCTGTTCTTGATGAGG-3'

P2X3 Sense 5'-ATCAACCGAGTAGTTCAGC-3' 695 
Antisense 5'-GATGCACTGGTCCCAGG-3'

P2X4 Sense 5'-GGCCTTCCTGTTCGAGTACG-3' 596

Antisense 5'-TGGGAAGGATATTCCTCTTGC-3'

P2X5 Sense 5'-CATGGGGCAGGCGGG-3' 598

Antisense 5'-GGGGAAACGGATGTGGTTCT-3'

P2X6 Sense 5'-AGTTCAACTTCTCTAAGTCCAATGC-3' 470

Antisense 5'-CTCTATCCACATACAGCAGTAGC-3'

(Continued on the next page)

SUPPLEMENTAL TABLE 1 (continued)

mRNA sequences size $(\mathrm{bp})$

P2X7 Sense 5'-CCCCGGCCACAACTACACCACGAGAAAC-3' 440

Antisense 5'-CCGAAGTAGGAGAGGGTTGAGCCGATG-3'

P2Y1 Sense 5'-CCTGCGAAGTTATTTCATCTA-3' 318

Antisense 5'-GTTGAGACTTGCTAGACCTCT-3'

P2Y2 Sense 5'-GCAGCATCCTCTTCCTCACCT-3' 502

Antisense 5'-CATGTTGATGGCGTTGAGGGT-3'

P2Y4 Sense 5'-GGCATTGTCAGACACCTTGTA-3' 551

Antisense 5'-AAGGCACGAAGCAGACAGCAA-3'

P2Y6 Sense 5'-CGCTTCCTCTTCTATGCCAA-3' 480

Antisense 5'-GTAGGCTGTCTTGGTGATGTG-3'

P2Y11 Sense 5'-ACTTCCTGTGGCCCATACTG-3' 499

Antisense 5'-GCTGTCCCCAGACACTTGAT-3'

P2Y12 Sense 5'-CTAAGATTCTCTCTGTTGTCATCTG-3' 432

Antisense 5'-ACAGAGTGCTCTCTTTCACATAG-3'

P2Y13 Sense 5'-TGTGTCGTTTTTCTTCGGTG-3' 578

Antisense 5'-TGCTGCCAAAAAGAGAGTTG-3'

P2Y14 Sense 5'-CGCAACATATTCAGCATCGTGT-3' 102

Antisense 5'-GCTGTAATGAGCTTCGGTCTGAC-3'

GAPDH Sense 5'-GCAGGGGGGAGCCAAAAGGG-3' 567

Antisense 5'-TGCCAGCCCCAGCGTCAAAG-3'

REFERENCES

1. Elieh Ali Komi D, Grauwet K. Role of Mast Cells in Regulation of T Cell Responses in Experimental and Clinical Settings. Clinic Rev Allerg Immunol. 2018;54(3):432-445.

2. Bais S, Kumari R, Prashar Y, Gill NS. Review of various molecular targets on mast cells and its relation to obesity: A future perspective. Diabetes Metab Syndr. 2017;11 Suppl 2:S1001-s1007. 
3. Yu KT, Lyall R, Jariwala N, Zilberstein A, Haimovich J. Antigen- and ionophore-induced signal transduction in rat basophilic leukemia cells involves protein tyrosine phosphorylation. J Biol Chem. 1991;266(33):22564-22568.

4. Schulman ES. The role of mast cell derived mediators in airway hyperresponsiveness. Chest. 1986;90(4):578-583.

5. Schulman ES. The role of mast cells in inflammatory responses in the lung. Critical Reviews in Immunology. $1993 ; 13(1): 35-70$.

6. Schulman ES, Adkinson NF, Jr., Newball HH. Cyclooxygenase metabolites in human lung anaphylaxis: airway vs. parenchyma. Journal of Applied Physiology: Respiratory, Environmental \& Exercise Physiology. 1982;53(3):589-595.

7. Schulman ES, Newball HH, Demers LM, Fitzpatrick FA, Adkinson NF, Jr. Anaphylactic release of thromboxane A2, prostaglandin D2, and prostacyclin from human lung parenchyma. American Review of Respiratory Disease. 1981;124(4):402-406.

8. Mohanty JG, Raible DG, McDermott LJ, Pelleg A, Schulman ES. Effects of purine and pyrimidine nucleotides on intracellular $\mathrm{Ca}^{2+}$ in human eosinophils: activation of purinergic P2Y receptors. Journal of Allergy and Clinical Immunology. 2001;107(5):849-855.

9. Nishi H, Pelleg A, Schulman ES. IgE Receptor-Mediated Histamine Release in Human Lung Mast Cells: Modulation by Purinergic Receptor Ligands. Ann Clin Lab Sci. 2016;46(5):463-469.

10. Schulman ES, Glaum MC, Post T, et al. ATP modulates anti-IgE-induced release of histamine from human lung mast cells. American Journal of Respiratory Cell and Molecular Biology. 1999;20(3):530-537.

11. Di Virgilio F, Vuerich M. Purinergic signaling in the immune system. Auton Neurosci. 2015;191:117-123.

12. Junger WG. Immune cell regulation by autocrine purinergic signalling. Nat Rev Immunol. 2011;11(3):201-212.

13. Burnstock G. Purine and pyrimidine receptors. Cell Mol Life Sci. 2007;64(12):1471-1483.

14. Cieslak M, Wojtczak ACP. Role of purinergic receptors in the Alzheimer's disease. Purinergic Signal. 2018;14(4):331-344.

15. Di Virgilio F, Dal Ben D, Sarti AC, Giuliani AL, Falzoni S. The P2X7 Receptor in Infection and Inflammation. Immunity. 2017;47(1):15-31.

16. Kimura Y, Inoue Y, Honda H. Further studies on rat mast cell degranulation by IgE-anti-IgE and the inhibitory effect of drugs related to cAMP. Immunology. 1974;26(5):983-988.

17. Gao ZG, Ding Y, Jacobson KA. UDP-glucose acting at P2Y14 receptors is a mediator of mast cell degranulation. Biochem Pharmacol. 2010;79(6):873-879.

18. Burnstock G. Purine and purinergic receptors. Brain Neurosci Adv. 2018;2:2398212818817494.

19. von Kügelgen I. Pharmacological profiles of cloned mammalian P2Y-receptor subtypes. Pharmacol Ther. 2006;110(3):415-432.

20. Niyonsaba F, Song P, Yue H, et al. Antimicrobial peptide derived from insulin-like growth factor-binding protein 5 activates mast cells via Mas-related G protein-coupled receptor X2. Allergy. 2020;75(1):203-207.

21. Niyonsaba F, Ushio H, Hara M, et al. Antimicrobial peptides human beta-defensins and cathelicidin LL-37 induce the secretion of a pruritogenic cytokine IL-31 by human mast cells. J Immunol. 2010;184(7):3526-3534.

22. Yanashima K, Chieosilapatham P, Yoshimoto E, Okumura K, Ogawa H, Niyonsaba F. Innate defense regulator IDR-1018 activates human mast cells through G protein-, phospholipase C-, MAPK- and NF-Bsensitive pathways. Immunol Res. 2017;65(4):920-931. 
23. Kirshenbaum AS, Akin $\mathrm{C}, \mathrm{Wu} \mathrm{Y}$, et al. Characterization of novel stem cell factor responsive human mast cell lines LAD 1 and 2 established from a patient with mast cell sarcoma/leukemia; activation following aggregation of FcepsilonRI or FcgammaRI. Leuk Res. 2003;27(8):677-682.

24. Nishi H, Arai H, Momiyama T. NCI-H295R, a Human Adrenal Cortex-Derived Cell Line, Expresses Purinergic Receptors Linked to $\mathrm{Ca}^{2+}$-Mobilization/Influx and Cortisol Secretion. PLOS ONE. 2013;8(8):e71022.

25. Alberto AV, Faria RX, de Menezes JR, et al. Role of P2 Receptors as Modulators of Rat Eosinophil Recruitment in Allergic Inflammation. PLoS One. 2016;11(1):e0145392.

26. Communi D, Robaye B, Boeynaems JM. Pharmacological characterization of the human P2Y11 receptor. Br J Pharmacol. 1999;128(6):1199-1206.

27. Gruenbacher G, Gander H, Rahm A, et al. The Human G Protein-Coupled ATP Receptor P2Y(11) Is Associated With IL-10 Driven Macrophage Differentiation. Front Immunol. 2019;10:1870.

28. Kuang Y, Liu H, Guo S, Wang Y, Zhang H, Qiao Y. The antagonist of P2Y11 receptor NF157 ameliorates oxidized LDL-induced vascular endothelial inflammation. Artif Cells Nanomed Biotechnol. 2019;47(1):18391845 .

29. Ullmann H, Meis S, Hongwiset D, et al. Synthesis and structure-activity relationships of suramin-derived P2Y11 receptor antagonists with nanomolar potency. J Med Chem. 2005;48(22):7040-7048.

30. Alsaleh NB, Persaud I, Brown JM. Silver Nanoparticle-Directed Mast Cell Degranulation Is Mediated through Calcium and PI3K Signaling Independent of the High Affinity IgE Receptor. PLoS One. 2016;11(12):e0167366.

31. Munoz I, Danelli L, Claver J, et al. Kinesin-1 controls mast cell degranulation and anaphylaxis through PI3K-dependent recruitment to the granular Slp3/Rab27b complex. J Cell Biol. 2016;215(2):203-216.

32. Huber M, Cato ACB, Ainooson GK, et al. Regulation of the pleiotropic effects of tissue-resident mast cells. J Allergy Clin Immunol. 2019.

33. Schulman ES, Anderson CL. Lung hypersensitivity responses: dissociation of human lung mast cell leukotriene C4 (LTC4) from histamine release. Progress in Clinical \& Biological Research. 1985;199:209220.

34. Nishida K, Wang L, Morii E, et al. Requirement of Gab2 for mast cell development and KitL/c-Kit signaling. Blood. 2002;99(5):1866-1869.

35. Nishida K, Yamasaki S, Hasegawa A, Iwamatsu A, Koseki H, Hirano T. Gab2, via PI-3K, regulates ARF1 in FcepsilonRI-mediated granule translocation and mast cell degranulation. J Immunol. 2011;187(2):932941.

36. Santos Mde S, Naal RM, Baird B, Holowka D. Inhibitors of PI(4,5)P2 synthesis reveal dynamic regulation of IgE receptor signaling by phosphoinositides in RBL mast cells. Mol Pharmacol. 2013;83(4):793-804.

37. Siraganian RP, Hazard KA. Mechanisms of mouse mast cell activation and inactivation for IgE-mediated histamine release. J Immunol. 1979;122(5):1719-1725.

38. Bekhite MM, Finkensieper A, Binas S, et al. VEGF-mediated PI3K class IA and PKC signaling in cardiomyogenesis and vasculogenesis of mouse embryonic stem cells. J Cell Sci. 2011;124(Pt 11):1819-1830.

39. Pritchard RA, Falk L, Larsson M, Leinders M, Sorkin LS. Different phosphoinositide 3-kinase isoforms mediate carrageenan nociception and inflammation. Pain. 2016;157(1):137-146.

40. Chalhoub N, Zhu G, Zhu X, Baker SJ. Cell type specificity of PI3K signaling in Pdk1- and Pten-deficient brains. Genes Dev. 2009;23(14):1619-1624. 
41. Lee-Rivera I, López E, Parrales A, Alvarez-Arce A, López-Colomé AM. Thrombin promotes the expression of Ccnd1 gene in RPE cells through the activation of converging signaling pathways. Exp Eye Res. $2015 ; 139: 81-89$.

42. Tsuchiya A, Kanno T, Nishizaki T. PI3 kinase directly phosphorylates Akt1/2 at Ser473/474 in the insulin signal transduction pathway. J Endocrinol. 2014;220(1):49-59.

43. Pelleg A, Schulman ES, Barnes PJ. Extracellular Adenosine 5'-Triphosphate in Obstructive Airway Diseases. Chest. 2016;150(4):908-915.

44. Pelleg A, Schulman ES, Barnes PJ. Adenosine 5'-triphosphate's role in bradycardia and syncope associated with pulmonary embolism. Respir Res. 2018;19(1):142.

45. Burnstock G, Brouns I, Adriaensen D, Timmermans JP. Purinergic signaling in the airways. Pharmacol Rev. 2012;64(4):834-868.

46. Polosa R. Adenosine-receptor subtypes: their relevance to adenosine-mediated responses in asthma and chronic obstructive pulmonary disease. Eur Respir J. 2002;20(2):488-496.

47. Wang M, Wisniewski A, Pavord I, Knox A, Tattersfield A. Comparison of three inhaled non-steroidal anti-inflammatory drugs on the airway response to sodium metabisulphite and adenosine 5'-monophosphate challenge in asthma. Thorax. 1996;51(8):799-804.

48. Prieto L, Gutiérrez V, Liñana J, Marín J. Bronchoconstriction induced by inhaled adenosine 5'monophosphate in subjects with allergic rhinitis. Eur Respir J. 2001;17(1):64-70.

49. Chavez J, Vargas MH, Martinez-Zuniga J, et al. Allergic sensitization increases the amount of extracellular ATP hydrolyzed by guinea pig leukocytes. Purinergic Signal. 2019;15(1):69-76.

50. Yoshida K, Ito M, Matsuoka I. Divergent regulatory roles of extracellular ATP in the degranulation response of mouse bone marrow-derived mast cells. Int Immunopharmacol. 2017;43:99-107.

51. Djukanovic R, Finnerty JP, Holgate ST. Wheal-and-flare responses to intradermally injected adenosine 5'-monophosphate, hypertonic saline, and histamine: comparison of atopic and nonatopic subjects. J Allergy Clin Immunol. 1989;84(3):373-378.

52. Le TT, Berg NK, Harting MT, Li X, Eltzschig HK, Yuan X. Purinergic Signaling in Pulmonary Inflammation. Front Immunol. 2019;10:1633.

53. van Heusden C, Button B, Anderson WH, et al. Inhibition of ATP hydrolysis restores airway surface liquid production in cystic fibrosis airway epithelia. Am J Physiol Lung Cell Mol Physiol. 2020;318(2):L356-1365.

54. Ivonnet P, Salathe M, Conner GE. Hydrogen peroxide stimulation of CFTR reveals an Epac-mediated, soluble AC-dependent cAMP amplification pathway common to GPCR signalling. Br J Pharmacol. 2015;172(1):173-184.

55. Schmidt M, Evellin S, Weernink PA, et al. A new phospholipase-C-calcium signalling pathway mediated by cyclic AMP and a Rap GTPase. Nat Cell Biol. 2001;3(11):1020-1024.

56. Springett GM, Kawasaki H, Spriggs DR. Non-kinase second-messenger signaling: new pathways with new promise. Bioessays. 2004;26(7):730-738. 